\title{
Intelligent Control and Monitoring Module for Uninterruptible Power Supply System
}

\author{
Andriy Palamar \\ ORCID 0000-0003-2162-9011 \\ Computer Systems and Networks Department \\ Ternopil Ivan Puluj National Technical University \\ Ternopil, Ukraine \\ palamar.andrij@gmail.com
}

\begin{abstract}
This paper proposes an intelligent control and monitoring module for industrial uninterruptible power supply systems. The structure scheme of the module based on the parallel connected of two microcontrollers with ARM architecture is designed. The software for the module is developed and implemented.
\end{abstract}

Keywords-control system, monitoring, microcontroller, uninterruptible power supply.

\section{INTRODUCTION}

Industrial uninterruptible power supply (UPS) systems are widely used in order to ensure high quality power supply and protection of electronic equipment from failure. One of the most important components of modern UPS is a computer information and measurement system [1].

In most UPS, the core of such system is a microprocessor or microcontroller that performs all of the monitoring and control functions. Quite often, UPS is used to provide guaranteed power to remote objects to which the operator does not have constant access, so there is a requirement for remote monitoring of the device and its electrical parameters.

In the software and hardware complex, which was developed by the author $[2,3]$, the process of data transfer to a PC for remote monitoring is implemented using the Ethernet protocol, which requires significant hardware and software resources of the microcontroller. On the other hand, the process of battery charge management also requires significant computing power, as it requires operations of analog-to-digital conversion of electrical signals, and rapid response to changes in measured data depending on the control mode.

The combination of these tasks creates a significant load on the computing power of the microcontroller, which in turn reduces the response rate to changes in the electrical parameters of the battery, which is critical during transients during battery charging. To solve this problem, this paper proposes design and implementation of a two-core information and measurement control system for an uninterruptible power supply system, which implements the principle of parallelization of tasks.

The purpose of the work is to increase the reliability and efficiency of the industrial UPS control module by using parallel operation of two 32-bit microcontrollers.

\section{The Structure OF THE Module}

The UPS control module implements functions of electric parameters measurement and battery charge management process, check a condition of alarm signals, provides an opportunity of archiving of the measured data, carries out transfer of the received information to the personal computer.

The schematic block diagram of the control and monitoring module for uninterruptible power supply system is shown in Fig. 1. The structure of the module contains:

- two microcontrollers (STM32F103 and AT91SAM7X512);

- conversion and amplification circuit for receiving input analog signals;

- circuit that is responsible for receiving digital input signals;

- non-volatile memory chip for storing received data;

- USB and Ethernet controllers for PC connection;

- keyboard and LCD for user interaction.

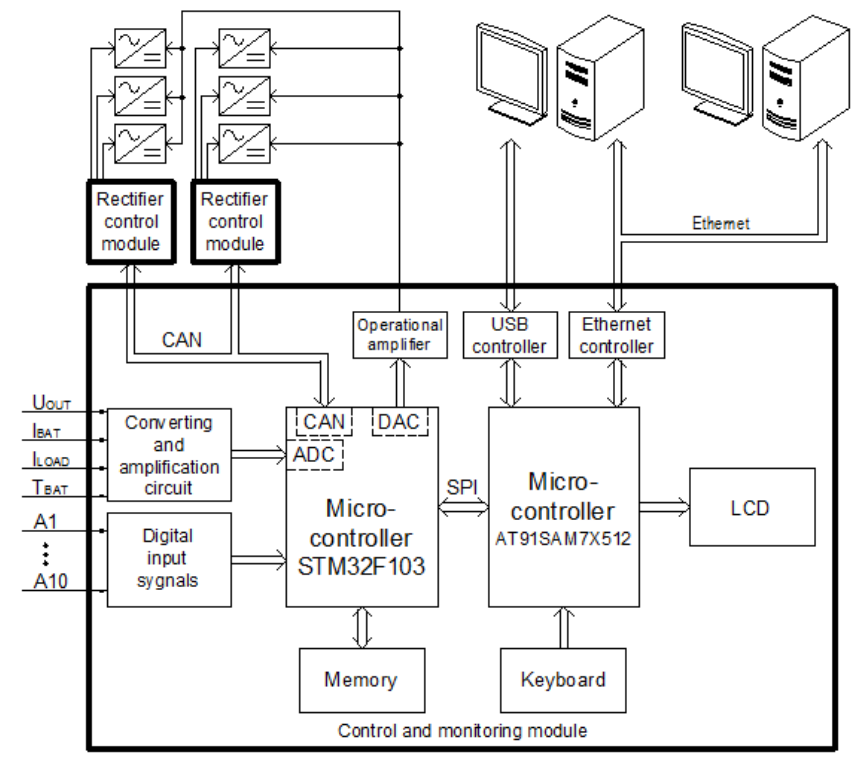

Fig. 1. Block diagram of the control and monitoring module for uninterruptible power supply system. 
Two 32-bit microcontrollers based on the ARM architecture provide basic control and monitoring functions of the UPS. The STM32F103 microcontroller contains builtin 16-channel 12-bit analog-to-digital converter (ADC) and two-channel digital-to-analog converter (DAC). It is responsible for performing tasks that require frequent checking of the system status and measuring its electrical parameters by using ADC. This microcontroller provides fast response to their change by generating an analog control signal for UPS rectifier modules using DAC. In addition, this microcontroller performs the function of controlling the operating modes of the USP such as battery charge process, residual capacity testing, etc.

The AT91SAM7X512 microcontroller with integrated Ethernet, CAN and USB interfaces is responsible to perform tasks that are not time-critical. For example, displaying of the measured data on the LCD display, reading the keyboard, transferring the information to the server for its storage in database and further processing. Serial peripheral interface (SPI) is used to provide fast and reliable data exchange process between these microcontrollers.

After the conversion and amplification, voltage, currents, and temperature signals are measured by using inner analogto-digital converter of the STM32F103 microcontroller. All deviations of the measured data from the normal values, alarms as well as time of an event are stored in a non-volatile memory.

The received data are visualized on the LCD on the front of the module. Electrical parameters of the system can be set or changed using the keyboard with four buttons. Digital signals of the UPS status are being read periodically by using the input output ports of the microcontroller. Real time clock is used to keep track of the current time.

A prototype of the designed control and monitoring module is illustrated in Fig. 2.

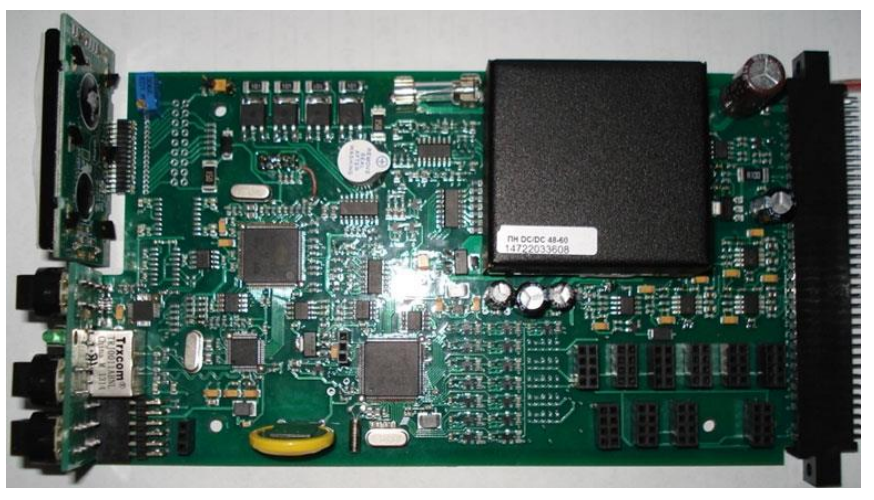

Fig. 2. A prototype of the control and monitoring module for an uninterruptible power supply.

\section{THE SOFTWARE OF THE MODULE}

The software of the control and monitoring system comprises of two parts. The first one is firmware for the microcontrollers. It is written by using $\mathrm{C}$ programming language. The second part of the software is a computer monitoring application - graphical user interface (Fig. 3).

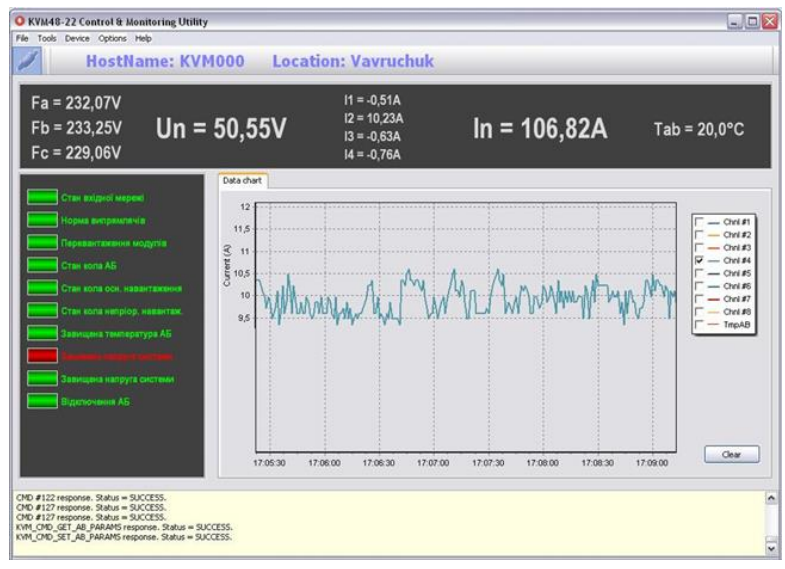

Fig. 3. Graphical user interface of UPS monitoring system for PC.

The main program of the microcontrollers includes the following subroutines: system initialization; data collection and processing; monitoring and display unit; control unit; data transfer unit.

The function of data collection and processing unit is to provide digitizing of the measured data and their previous processing. Information required to be collected includes: output voltage; battery current; load current; battery temperature. These data are used for management purposes of the control system.

The monitoring and display unit is designed to visualize the measured information on the LCD in a real-time and to provide access to archived data. Furthermore, it allows one to set and to modify parameters of the system.

Data transfer unit is responsible for transmitting the information to the computer through the Ethernet or USB interface. The amount of data to be transferred includes: the measured information, electrical parameters of the system, and the archived data from the memory of the module.

\section{CONCLUSIONS}

In this paper an intelligent control and monitoring module for UPS system was designed and implemented. The results of experimental tests of the developed module shows that the technology of parallelization of tasks by using two microcontroller allows to increase the reliability and efficiency of the control system for an uninterruptible power supply.

\section{REFERENCES}

[1] T. Addabbo, A. Fort, M. Mugnaini, V. Vignoli. "Distributed UPS control systems reliability analysis," Measurement, 2017, Vol. 110, pp. 275-283. doi: 10.1016/j.measurement.2017.06.021

[2] A. Palamar, M. Karpinskyy. "Control of an uninterruptible power supply in a DC microgrid system," 10th International Symposium Symposium "Topical Problems in the Field of Electrical and Power Engineering" and "Doctoral School of Energy and Geotechnology II", Pärnu, Estonia. 2011, pp. 80-84.

[3] A. Palamar, M. Karpinskyy, V. Vodovozov. "Design and implementation of a digital control and monitoring system for an AC/DC UPS," 7th International Conference-Workshop "Compatibility and Power Electronics" CPE 2011, Tallinn, Estonia. 2011, pp. 173-177. doi: 10.1109/CPE.2011.5942227 